\title{
Omega-3 fatty acids and incident type 2 diabetes: a systematic review and meta-analysis
}

\author{
Jason H. Y. Wu ${ }^{1,2 *}$, Renata Micha ${ }^{1}$, Fumiaki Imamura ${ }^{1}$, An Pan ${ }^{3}$, Mary L. Biggs ${ }^{4}$, Owais Ajaz ${ }^{5,6}$, \\ Luc Djousse $^{5,6}$, Frank B. Hu ${ }^{1,3,7}$ and Dariush Mozaffarian ${ }^{1,3,7,8}$ \\ ${ }^{1}$ Department of Epidemiology, Harvard School of Public Health, Boston, 665 Huntington Ave, Kresge Building-913, \\ Boston MA 02115, USA \\ ${ }^{2}$ School of Medicine and Pharmacology, University of Western Australia, Perth, Australia \\ ${ }^{3}$ Department of Nutrition, Harvard School of Public Health, Boston, MA, USA \\ ${ }^{4}$ Department of Biostatistics, University of Washington, Seattle, USA \\ ${ }^{5}$ Divisions of Aging, Department of Medicine, Brigham and Women's Hospital and Harvard Medical School, MA, USA \\ ${ }^{6}$ Massachusetts Veterans Epidemiology and Research Information Center, Boston Veterans Affairs Healthcare System, Boston, \\ MA, USA \\ ${ }^{7}$ Channing Laboratory, Department of Medicine, Brigham and Women's Hospital and Harvard Medical School, Boston, MA, USA \\ ${ }^{8}$ Division of Cardiovascular Medicine, Department of Medicine, Brigham and Women's Hospital and Harvard Medical \\ School, Boston, MA, USA
}

\section{Abstract}

The relationship between omega-3 polyunsaturated fatty acids ( $n$-3 PUFA) from seafood sources (eicosapentaenoic acid, EPA; docosahexaenoic acid, DHA) or plant sources (alpha-linolenic acid, ALA) and risk of type 2 diabetes mellitus (DM) remains unclear. We systematically searched multiple literature databases through June 2011 to identify prospective studies examining relations of dietary $n$-3 PUFA, dietary fish and/or seafood, and circulating $n$-3 PUFA biomarkers with incidence of DM. Data were independently extracted in duplicate by 2 investigators, including multivariate-adjusted relative risk (RR) estimates and corresponding 95\% CI. Generalized least-squares trend estimation was used to assess dose-response relationships, with pooled summary estimates calculated by both fixed-effect and random-effect models. From 288 identified abstracts, 16 studies met inclusion criteria, including 18 separate cohorts comprising 540184 individuals and 25670 cases of incident DM. Consumption of fish and/or seafood was not significantly associated with DM $(n=13$ studies; RR per $100 \mathrm{~g} / \mathrm{d}=1 \cdot 12,95 \% \mathrm{CI}=0 \cdot 94,1 \cdot 34)$; nor were consumption of EPA+DHA $(n=16$ cohorts; RR per $250 \mathrm{mg} / \mathrm{d}=1 \cdot 04,95 \%$ $\mathrm{CI}=0.97,1 \cdot 10)$ nor circulating levels of EPA+DHA biomarkers $(n=5$ cohorts; RR per $3 \%$ of total fatty acids $=0.94,95 \% \mathrm{CI}=0.75$, 1.17). Both dietary ALA ( $n=7$ studies; RR per $0.5 \mathrm{~g} / \mathrm{d}=0.93,95 \% \mathrm{CI}=0.83,1 \cdot 04)$ and circulating ALA biomarker levels $(n=6$ studies; RR per $0 \cdot 1 \%$ of total fatty acid $=0 \cdot 90,95 \% \mathrm{CI}=0 \cdot 80,1 \cdot 00, P=0 \cdot 06)$ were associated with non-significant trend towards lower risk of DM. Substantial heterogeneity $\left(I^{2} \sim 80 \%\right)$ was observed among studies of fish/seafood or EPA+DHA and DM; moderate heterogeneity $(<55 \%)$ was seen for dietary and biomarker ALA and DM. In unadjusted meta-regressions, study location (Asia vs. North America/ Europe), mean BMI, and duration of follow-up each modified the association between fish/seafood and EPA+DHA consumption and DM risk ( $P$-interaction $\leq 0.02 \mathrm{each}$ ). We had limited statistical power to determine the independent effect of these sources of heterogeneity due to their high collinearity. The overall pooled findings do not support either major harms or benefits of fish/seafood or EPA+DHA on development of DM, and suggest that ALA may be associated with modestly lower risk. Reasons for potential heterogeneity of effects, which could include true biologic heterogeneity, publication bias, or chance, deserve further investigation.

Key words: omega-3 fatty acid: type 2 diabetes: systematic review: meta-analysis: prospective cohort

\section{Introduction}

Type 2 diabetes mellitus (DM) accounts for $90-95 \%$ of all diabetes cases and has reached epidemic proportions globally, including in both developed and developing countries ${ }^{(1)}$. As a major risk factor for coronary heart disease, stroke, blindness, kidney failure, and peripheral arterial disease, DM poses tremendous public health burdens. Epidemiological and clinical trial evidence demonstrate that lifestyle including diet plays a major role in the development of $\mathrm{DM}^{(2)}$. Further understanding of the role of specific foods and nutrients in the pathogenesis of DM is of paramount importance.

Omega-3 polyunsaturated fatty acids ( $n-3$ PUFA) include eicosapentaenoic acid (EPA, 20:5n-3) and docosahexaenoic acid (DHA, 22:6n-3) from seafood, and alpha-linolenic acid 
(ALA, 18:3n-3) from plant sources. Based on animal experimental studies, $n$-3 PUFA improve several metabolic abnormalities underlying the development of DM. Such effects include insulin-sensitizing effects via increased production and secretion of adipocytokines such as adiponectin and leptin ${ }^{(3-6)}$; and potential prevention of insulin resistance via anti-inflammatory effects mediated directly ${ }^{(7)}$ or through conversion to specialized pro-resolution mediators such as resolvins and protectins ${ }^{(8,9)}$. Through modulation of transcription factors (e.g. sterol regulatory element binding protein-1c), n-3 PUFA could also enhance fatty acid oxidation and reduce de novo lipogenesis, effects which could reduce hepatic fat accumulation and preserve hepatic insulin sensitivity ${ }^{(10-13)}$.

Despite metabolic benefits in animal experiments, the impact of $n-3$ PUFA consumption on risk of DM in humans remain uncertain. In meta-analyses of controlled supplementation trials, $n$-3 PUFA supplementation does not produce major changes in biomarkers of glucose-insulin homeostasis in subjects with $\mathrm{DM}^{(14-16)}$; similar trials in healthy subjects have reported conflicting findings ${ }^{(17)}$. In addition to these short-term trials, which generally tested high supplemental doses of $n$-3 PUFA, several long-term prospective studies have assessed how habitual dietary consumption of $n-3$ PUFA or seafood, or circulating biomarkers of consumption, relate to incidence of DM, but with mixed findings. Therefore, whether $n$-3 PUFA influence risk of incident DM and, if so, the direction and magnitude of effect remain unknown. To address these important scientific and public health questions, we carried out a systematic review and meta-analysis of prospective studies that assessed the relation of dietary $n-3$ PUFA, fish and/or seafood consumption, and biomarker levels of $n-3$ PUFA with the incidence of DM.

\section{Methods}

\section{Search strategy and eligibility criteria}

We followed the Meta-analysis of Observational Studies in Epidemiology guidelines for the design, implementation, analysis and reporting of this study ${ }^{(18)}$. We searched for all prospective cohort studies, including nested prospective studies, that assessed the association of dietary $n-3$ PUFA intake (ALA, EPA+DHA, or EPA and DHA individually), dietary fish and/or seafood intake, and biomarkers of $n-3$ PUFA (ALA, EPA+DHA, or EPA and DHA individually) with incidence of DM. Searches were performed electronically through MEDLINE, EMBASE, Latin American and Caribbean Health Sciences Literature (LILACS), related articles, hand-searching of references, and direct author contact. Key words included (among others) omega-3, alpha-linolenic acid, eicosapentaenoic acid, docosahexaenoic acid, fish oils, fishes, diabetes mellitus, cohort studies, prospective studies, and nested case control; the full search terms are available on request. Searches included the earliest available online indexing year through to June 30, 2011, with no language restrictions.

Studies were eligible for inclusion if they were prospective cohort studies that provided a multivariate-adjusted effect estimate (odds ratio, relative risk [RR], or hazard ratio) and information about its variance for any of the exposures of interest and incident DM. Exclusion criteria included studies of pregnant women or children $(<19 \mathrm{y})$, studies of type 1 diabetes, retrospective case-control studies, cross-sectional and ecological studies, literature reviews, commentaries, editorials, letters, case reports, studies which investigated only shellfish or selected subtypes of fish intake (e.g., fried fish), and studies which provided only crude risk estimates. If multiple manuscripts were published from the same cohort, we included the most up-to-date analyses that had accrued the highest number of DM cases.

\section{Selection of articles}

The titles and abstracts of all identified articles were screened by one investigator ( J. W.) for eligibility. Two investigators (J. W., R. M.) assessed independently and in duplicate the full texts of the remaining articles to reach a final decision on inclusion or exclusion, with differences resolved by consensus. Of 288 initially identified articles, 262 were excluded based on title and abstract (Fig. 1). Of 26 full text articles, 10 were excluded because they were duplicate publications from the same cohort $(n=3)^{(19-21)}$, assessed consumption of restaurant fried fish intake but not overall fish intake $(n=1)^{(22)}$, lacked RR estimates for fish or $n$-3 PUFA exposures $(n=4)^{(23-26)}$, or did not separately report results for DM vs. impaired glucose tolerance $(n=2)^{(27,28)}$. After final exclusions, 16 studies were identified for inclusion in the meta-analysis ${ }^{(29-44)}$

\section{Data extraction}

For each included study, data were extracted independently and in duplicate by two investigators (J. W., R. M.) using a standardized electronic form, including information on study design, study location (North America, Europe, or Asia/Australia), whether the analysis was pre-specified or post hoc, subject inclusion and exclusion criteria, sample size, subject age, body mass index (BMI), co-morbidities, gender, race, duration of follow-up (mean, median or max number of years), number of events, methods for dietary assessment and diagnosis of DM, laboratory procedure for fatty acid biomarkers (e.g. plasma phospholipid measurements), covariates adjusted for, and multivariable-adjusted risk estimates including data needed to calculate its variance (e.g., CI, SE, or $P$-value). For studies reporting risk across categories of exposure, we further recorded for each study the exposure-category-specific data on person-years of follow up, number of subjects, number of DM cases, median value of exposures, and risk estimate including its variance. For studies reporting on both fish and total seafood intake, we extracted both estimates. When more than one multivariable model was assessed, we extracted risk estimates having the greatest adjustment for potential confounders that did not also include potential intermediates (blood glucose, triglyceride, or inflammatory biomarkers). Whereas no single accepted criteria for grading quality of cohort exists, such grading can be useful for exploring quality and heterogeneity. We assessed quality using previously reported methods by considering 5 criteria: 


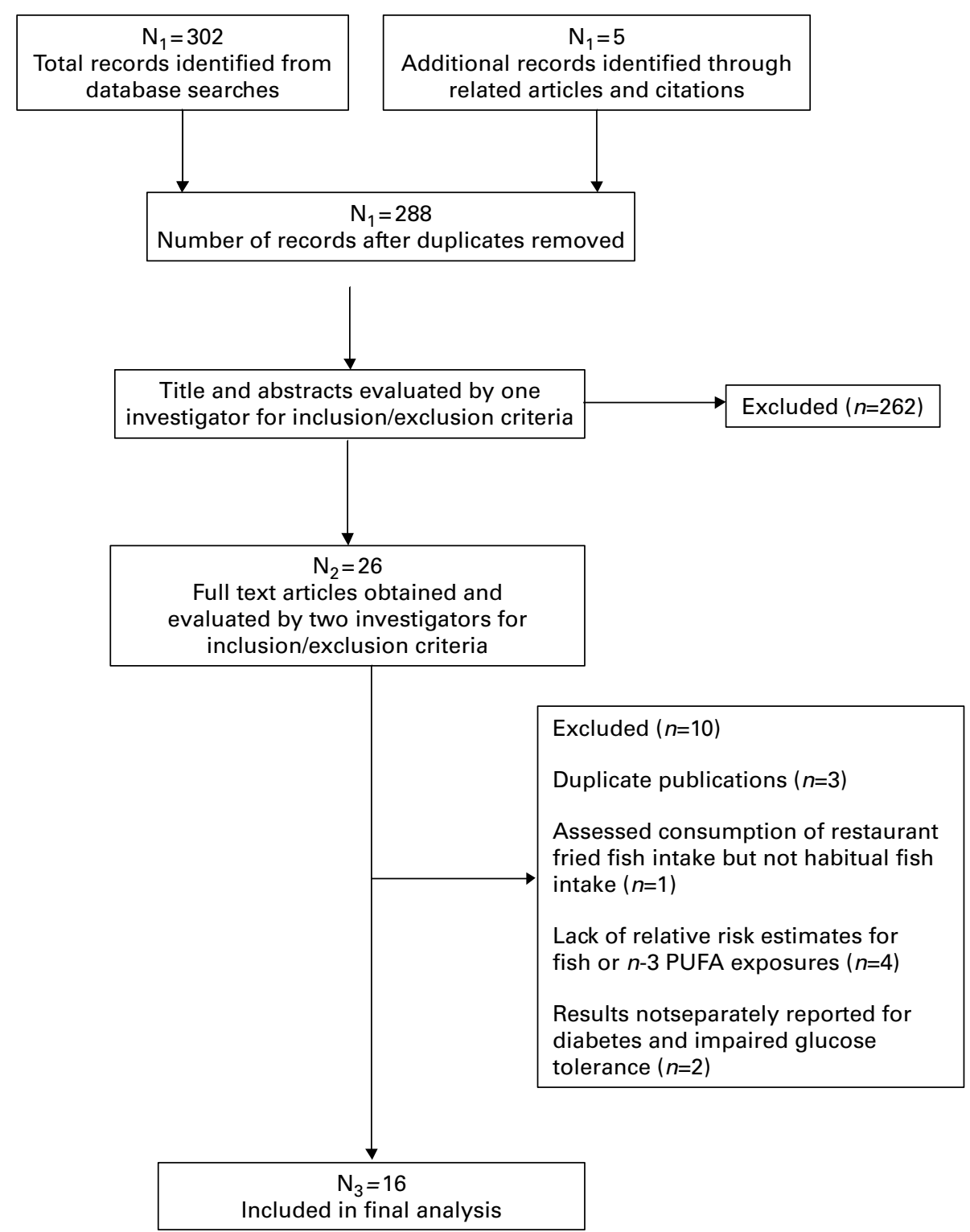

Fig. 1. Search, screening, and selection process of prospective cohort studies of dietary $n-3$ PUFA, fish/seafood, and circulating $n-3$ PUFA biomarkers and risk of type 2 diabetes.

appropriateness and reporting of inclusion and exclusion criteria, methods for assessment of exposure, methods for assessment of outcome, adjustment for confounding, and evidence of bias ${ }^{(45)}$.

Among the 16 studies included in the meta-analysis, we contacted and received responses from authors of 11 studies for relevant missing information, such as baseline characteristics (age, BMI, exposure distributions) or exposure category data (number of participants, person-years of follow-up, number of cases, median level of exposure, or risk estimates and $95 \% \mathrm{CI}$ ).

\section{Statistical analysis}

Because nearly all studies reported risk across exposure categories, risk estimates were meta-analyzed using the 2-step generalized least-squares trend (GLST) model ${ }^{(46,47)}$. This method utilizes all information from all exposure categories to estimate the log-linear dose-response slope within each study, which are then pooled to derive an overall risk estimate. For studies already reporting risk estimates for linear differences in exposure (e.g., per SD change in biomarker levels $\left.{ }^{(34)}\right)$ rather than in categories, data were added to the GLST model at the second stage. Because DM events were relatively rare in all studies, we considered odds ratios and hazard ratios to approximate RR. Necessary data for the first step of GLST included, for each exposure category in each study, the multivariable-adjusted risk estimate and its corresponding standard errors, person-years of follow-up (for prospective cohort studies) or number of subjects (for nested case-cohort studies), median level of exposure, and number of cases in each exposure category. When category-specific 
Table 1. Characteristics of the identified 16 studies including 18 prospective cohorts that evaluated dietary $n-3$ PUFA, fish/seafood consumption, or biomarker $n$-3 PUFA levels and incidence of type 2 diabetes* $^{*}$

\begin{tabular}{|c|c|c|c|c|c|c|c|c|c|c|c|}
\hline $\begin{array}{l}\text { Author } \\
\text { (Year) }\end{array}$ & $\begin{array}{l}\text { Study name } \\
\text { (Country) }\end{array}$ & $\begin{array}{l}\text { Total N/ } \\
\text { number of } \\
\text { DM cases }\end{array}$ & $\begin{array}{l}\text { Mean } \\
\text { Age, y }\end{array}$ & $\begin{array}{l}\text { Mean } \\
\mathrm{BMI}, \\
\mathrm{kg} / \mathrm{m}^{2}\end{array}$ & $\begin{array}{l}\text { Men, } \\
\%\end{array}$ & $\begin{array}{l}\text { Follow-up } \\
\text { years }\end{array}$ & Exposures $\dagger$ & $\begin{array}{l}\text { Diabetes } \\
\text { ascertainment }\end{array}$ & Adjustmentł & $\begin{array}{l}\text { Quality } \\
\text { score§ }\end{array}$ & $\begin{array}{l}\text { Additional data } \\
\text { requested// } \\
\text { provided\| }\end{array}$ \\
\hline $\begin{array}{l}\text { Meyer } \\
\quad(2001)^{(36)}\end{array}$ & $\begin{array}{l}\text { lowa Women's Health } \\
\text { Study (USA) }\end{array}$ & $35988 / 1890$ & 62 & $26 \cdot 9$ & 0 & Max:11 & $\begin{array}{l}\text { Diet } \\
\quad \mathrm{EPA}+\mathrm{DHA}\end{array}$ & $\begin{array}{l}\text { Self-report. Validated } \\
\text { in sub-cohort by } \\
\text { physician diagnosis }\end{array}$ & +++ & 3 & Yes/No \\
\hline $\begin{array}{l}\text { van Dam } \\
(2002)^{(40)}\end{array}$ & $\begin{array}{l}\text { Health Professional } \\
\text { Follow-up Study } \\
\text { (HPFS) (USA) }\end{array}$ & $42504 / 1321$ & 54 & $25 \cdot 5$ & 100 & Mean: 11 & $\begin{array}{l}\text { Diet } \\
\text { ALA }\end{array}$ & $\begin{array}{l}\text { Self-report confirmed by } \\
\text { supplemental } \\
\text { questionnaire }\end{array}$ & +++ & 5 & No/- \\
\hline Wang $(2003)^{(44)}$ & $\begin{array}{l}\text { Atherosclerosis Risk in } \\
\text { Communities Study } \\
\text { (USA) }\end{array}$ & $2909 / 252$ & 54 & $26 \cdot 7$ & $46 \cdot 9$ & Mean: $8 \cdot 1$ & $\begin{array}{l}\text { Biomarker } \\
\text { ALA }\end{array}$ & $\begin{array}{l}\text { Self-reported diabetes } \\
\text { medication use or } \\
\text { physician diagnosis or } \\
\text { fasting or non fasting } \\
\text { glucose }\end{array}$ & ++ & 4 & Yes/Yes \\
\hline $\begin{array}{l}\text { Hodge } \\
\qquad(2007)^{(32)}\end{array}$ & $\begin{array}{l}\text { Melbourne Collaborative } \\
\text { Cohort Study } \\
\text { (Australia) }\end{array}$ & १3737/346 & 55 & 27 & $44 \cdot 1$ & Mean: $4 \cdot 1$ & $\begin{array}{l}\text { Diet } \\
\text { ALA } \\
\text { EPA } \\
\text { DHA } \\
\text { EPA+DHA } \\
\text { Biomarker } \\
\text { ALA } \\
\text { EPA } \\
\text { DHA } \\
\text { EPA+DHA }\end{array}$ & $\begin{array}{l}\text { Self-report confirmed by } \\
\text { subject's physician. }\end{array}$ & ++ & 4 & Yes/Yes \\
\hline $\begin{array}{l}\text { Krachler } \\
\qquad(2008)^{(34)}\end{array}$ & $\begin{array}{l}\text { Vasterbotten Intervention } \\
\text { Program (Sweden) }\end{array}$ & $9450 / 159$ & 52 & 27 & $58 \cdot 4$ & Mean: 8.8 & $\begin{array}{l}\text { Biomarker } \\
\text { ALA } \\
\text { EPA } \\
\text { DHA } \\
\text { EPA+DHA }\end{array}$ & $\begin{array}{l}\text { Subjects attended health } \\
\text { exam at local primary } \\
\text { care center, diabetes } \\
\text { confirmed according } \\
\text { to WHO } 1998 \text { criteria. }\end{array}$ & ++ & 4 & Yes/Yes \\
\hline Vang $(2008)^{(42)}$ & $\begin{array}{l}\text { Adventist Mortality Study } \\
\text { and Adventist Health } \\
\text { Study (USA) }\end{array}$ & $8401 / 531$ & 65 & 24.5 & 61 & Mean: 16 & $\begin{array}{l}\text { Diet } \\
\quad \text { Seafood } \\
\text { EPA+DHA** }\end{array}$ & Self-report. & + & 1 & Yes/No \\
\hline $\begin{array}{l}\text { Kaushik } \\
\qquad(2009)^{(33)}\end{array}$ & $\begin{array}{l}\text { Nurses' Health Study } \\
\text { (NHS) (USA) }\end{array}$ & $61031 / 4159$ & 52 & $24 \cdot 9$ & 0 & Mean: $16 \cdot 7$ & $\begin{array}{l}\text { Diet } \\
\text { Fish } \\
\text { EPA+DHA }\end{array}$ & $\begin{array}{l}\text { Self-report confirmed by } \\
\text { supplementary } \\
\text { questionnaire. }\end{array}$ & +++ & 5 & No/No \\
\hline $\begin{array}{l}\text { Kaushik } \\
\qquad(2009)^{(33)}\end{array}$ & $\begin{array}{l}\text { Nurses' Health Study } \\
\text { 2(NHS2) (USA) }\end{array}$ & $91669 / 2728$ & 36 & $24 \cdot 5$ & 0 & Mean: $13 \cdot 7$ & $\begin{array}{l}\text { Diet } \\
\text { Fish } \\
\text { EPA+DHA }\end{array}$ & $\begin{array}{l}\text { Self-report confirmed by } \\
\text { supplementary } \\
\text { questionnaire. }\end{array}$ & +++ & 5 & No/No \\
\hline $\begin{array}{l}\text { Kaushik } \\
\qquad(2009)^{(33)}\end{array}$ & $\begin{array}{l}\text { Health Professional } \\
\text { Follow-up Study } \\
\text { (HPFS) (USA) }\end{array}$ & $42504 / 2493$ & 53 & 24.9 & 100 & Mean: $16 \cdot 0$ & $\begin{array}{l}\text { Diet } \\
\quad \text { Fish } \\
\text { EPA+DHA }\end{array}$ & $\begin{array}{l}\text { Self-report confirmed by } \\
\text { supplementary } \\
\text { questionnaire. }\end{array}$ & +++ & 5 & No/No \\
\hline Patel $(2009)^{(39)}$ & EPIC-Norfolk (UK) & $21984 / 725$ & 58 & $26 \cdot 3$ & $44 \cdot 6$ & Median: $10 \cdot 2$ & $\begin{array}{l}\text { Diet } \\
\quad \text { Seafood } \\
\text { EPA+DHA }\end{array}$ & $\begin{array}{l}\text { Self-report confirmed by } \\
\text { linkage with health } \\
\text { registries or cases } \\
\text { detected by linkage to } \\
\text { health registries alone. }\end{array}$ & +++ & 5 & Yes/Yes \\
\hline $\begin{array}{l}\text { van Wouden- } \\
\text { bergh } \\
(2009)^{(41)}\end{array}$ & $\begin{array}{l}\text { Rotterdam study } \\
\text { (Netherlands) }\end{array}$ & $4472 / 463$ & 67 & $26 \cdot 2$ & 41 & Median: $12 \cdot 4$ & $\begin{array}{l}\text { Diet } \\
\text { Fish } \\
\text { EPA } \\
\text { DHA } \\
\text { EPA+DHA }\end{array}$ & $\begin{array}{l}\text { Diabetes status } \\
\text { confirmed by general } \\
\text { practitioners according } \\
\text { to WHO } 1999 \text { criteria. }\end{array}$ & +++ & 4 & Yes/Yes \\
\hline
\end{tabular}




\section{Nes British Journal of Nutrition}

Table 1. Continued

\begin{tabular}{|c|c|c|c|c|c|c|c|c|c|c|c|}
\hline $\begin{array}{l}\text { Author } \\
\text { (Year) }\end{array}$ & $\begin{array}{l}\text { Study name } \\
\text { (Country) }\end{array}$ & $\begin{array}{l}\text { Total N/ } \\
\text { number of } \\
\text { DM cases }\end{array}$ & $\begin{array}{l}\text { Mean } \\
\text { Age, y }\end{array}$ & $\begin{array}{l}\text { Mean } \\
\mathrm{BMl} \\
\mathrm{kg} / \mathrm{m}^{2}\end{array}$ & $\begin{array}{l}\text { Men, } \\
\%\end{array}$ & $\begin{array}{l}\text { Follow-up } \\
\text { years }\end{array}$ & Exposures† & $\begin{array}{l}\text { Diabetes } \\
\text { ascertainment }\end{array}$ & Adjustment‡ & $\begin{array}{l}\text { Quality } \\
\text { score§ }\end{array}$ & $\begin{array}{l}\text { Additional data } \\
\text { requested// } \\
\text { provided\| }\end{array}$ \\
\hline Patel $(2010)^{(38)}$ & EPIC-Norfolk (UK) & ๆ 383/199 & 64 & $28 \cdot 1$ & $53 \cdot 3$ & Mean: $10 \cdot 3$ & $\begin{array}{l}\text { Diet } \\
\text { ALA } \\
\text { EPA } \\
\text { DHA } \\
\text { Biomarker } \\
\text { ALA } \\
\text { EPA } \\
\text { DHA } \\
\text { EPA+DHA }\end{array}$ & $\begin{array}{l}\text { Self-report confirmed by } \\
\text { linkage with health } \\
\text { registries. }\end{array}$ & ++ & 4 & Yes/Yes \\
\hline $\begin{array}{l}\text { Brostow } \\
\qquad(2011)^{(29)}\end{array}$ & $\begin{array}{l}\text { Singapore Chinese } \\
\text { Health Study } \\
\text { (Singapore) }\end{array}$ & $43176 / 2252$ & 55 & 23 & $42 \cdot 4$ & Mean: $5 \cdot 7$ & $\begin{array}{l}\text { Diet } \\
\text { Fish } \\
\text { Seafood } \\
\text { ALA } \\
\text { EPA+DHA }\end{array}$ & $\begin{array}{l}\text { Self-report confirmed by } \\
\text { linkage with hospital } \\
\text { records or supplemen- } \\
\text { tary questionnaire }\end{array}$ & +++ & 5 & Yes/Yes \\
\hline $\begin{array}{l}\text { Djousse } \\
\qquad(2011)^{(31)}\end{array}$ & $\begin{array}{l}\text { Women's Health Study } \\
\text { (WHS) (USA) }\end{array}$ & $36328 / 2370$ & 55 & $25 \cdot 9$ & 0 & Mean: $12 \cdot 4$ & $\begin{array}{l}\text { Diet } \\
\text { Fish } \\
\text { ALA } \\
\text { EPA } \\
\text { DHA } \\
\text { EPA+DHA }\end{array}$ & $\begin{array}{l}\text { Self-report confirmed by } \\
\text { telephone interview or } \\
\text { supplemental } \\
\text { questionnaire }\end{array}$ & +++ & 5 & Yes/Yes \\
\hline $\begin{array}{l}\text { Djousse } \\
\qquad(2011)^{(30)}\end{array}$ & $\begin{array}{l}\text { Cardiovascular Health } \\
\text { Study (CHS) (USA) }\end{array}$ & $3088 / 204$ & 75 & $26 \cdot 4$ & $38 \cdot 9$ & Mean: $9 \cdot 6$ & $\begin{array}{l}\text { Diet } \\
\text { Fish } \\
\text { ALA } \\
\text { EPA+DHA } \\
\text { Biomarker } \\
\text { ALA } \\
\text { EPA } \\
\text { DHA } \\
\text { EPA+DHA }\end{array}$ & $\begin{array}{l}\text { Diabetes medication } \\
\text { use, or fasting or } \\
\text { nonfasting glucose } \\
\text { level }\end{array}$ & +++ & 4 & Yes/Yes \\
\hline $\begin{array}{l}\text { Kroger } \\
\qquad(2011)^{(35)}\end{array}$ & $\begin{array}{l}\text { EPIC-Potsdam } \\
\text { (Germany) }\end{array}$ & $2724 / 673$ & 51 & $26 \cdot 9$ & 43.5 & Mean: $6 \cdot 3$ & $\begin{array}{l}\text { Diet } \\
\text { ALA } \\
\text { EPA } \\
\text { DHA } \\
\text { EPA+DHA } \\
\text { Biomarker } \\
\text { ALA } \\
\text { EPA } \\
\text { DHA } \\
\text { EPA+DHA }\end{array}$ & $\begin{array}{l}\text { Self-report confirmed by } \\
\text { diagnosing physician }\end{array}$ & +++ & 4 & Yes/Yes \\
\hline Nanri $(2011)^{(37)}$ & JPHC-Men (Japan) & $22921 / 572$ & 56 & $23 \cdot 6$ & 100 & Mean: 5 & $\begin{array}{l}\text { Diet } \\
\text { Seafood } \\
\text { EPA+DHA** }\end{array}$ & $\begin{array}{l}\text { Self-report validated by } \\
\text { medical records in } \\
\text { sub-group }\end{array}$ & +++ & 5 & No/- \\
\hline Nanri $(2011)^{(37)}$ & JPHC-Women (Japan) & $29759 / 399$ & 56 & 23.4 & 0 & Mean: 5 & $\begin{array}{l}\text { Diet } \\
\text { Seafood } \\
\text { EPA+DHA }\end{array}$ & $\begin{array}{l}\text { Self-report validated by } \\
\text { medical records in } \\
\text { sub-group }\end{array}$ & +++ & 5 & No/- \\
\hline $\begin{array}{l}\text { Villegas } \\
\qquad(2011)^{(43)}\end{array}$ & $\begin{array}{l}\text { Shanghai Men's Health } \\
\text { Study (SMHS) (China) }\end{array}$ & $51963 / 900$ & 54 & $23 \cdot 6$ & 100 & Mean: 4 & $\begin{array}{l}\text { Diet } \\
\quad \text { Fish } \\
\text { Seafood } \\
\text { EPA+DHA }\end{array}$ & $\begin{array}{l}\text { Self-report confirmed by } \\
\text { diabetes medication } \\
\text { use or fasting glucose } \\
\text { or OGTT. }\end{array}$ & +++ & 5 & Yes/Yes \\
\hline
\end{tabular}




\section{N British Journal of Nutrition}

Table 1. Continued

\begin{tabular}{|c|c|c|c|c|c|c|c|c|c|c|c|}
\hline $\begin{array}{l}\text { Author } \\
\text { (Year) }\end{array}$ & $\begin{array}{l}\text { Study name } \\
\text { (Country) }\end{array}$ & $\begin{array}{l}\text { Total } \mathrm{N} / \\
\text { number of } \\
\text { DM cases }\end{array}$ & $\begin{array}{l}\text { Mean } \\
\text { Age, y }\end{array}$ & $\begin{array}{l}\text { Mean } \\
\mathrm{BMl} \\
\mathrm{kg} / \mathrm{m}^{2}\end{array}$ & $\begin{array}{l}\text { Men, } \\
\%\end{array}$ & $\begin{array}{l}\text { Follow-up } \\
\text { years }\end{array}$ & Exposures $\dagger$ & $\begin{array}{l}\text { Diabetes } \\
\text { ascertainment }\end{array}$ & Adjustmentł & $\begin{array}{l}\text { Quality } \\
\text { score§ }\end{array}$ & $\begin{array}{l}\text { Additional data } \\
\text { requested/ } \\
\text { provided\| }\end{array}$ \\
\hline $\begin{array}{l}\text { Villegas } \\
\qquad(2011)^{(43)}\end{array}$ & $\begin{array}{l}\text { Shanghai Women's } \\
\text { Health Study (SWHS) } \\
\text { (China) }\end{array}$ & 64 193/3034 & 51 & $23 \cdot 8$ & 0 & Mean: 8.9 & $\begin{array}{l}\text { Diet } \\
\quad \text { Fish } \\
\text { Seafood } \\
\text { EPA+DHA }\end{array}$ & $\begin{array}{l}\text { Self-report confirmed by } \\
\text { diabetes medication } \\
\text { use or fasting glucose } \\
\text { or OGTT. }\end{array}$ & +++ & 5 & Yes/Yes \\
\hline
\end{tabular}

EPIC, European Prospective Investigation into Cancer and Nutrition; JPHC, Japan Public Health Center-based Prospective Study; Fish, refers to finfish; Seafood, refers to finfish and shellfish combined.

*All exposure-type 2 diabetes risk assessments were pre-specified primary analyses, except dietary fish $/ n-3$ PUFA intake in Djousse et al ${ }^{(30)}$, which were secondary analyses.

tDietary exposures were all assessed using food frequency questionnaires; fatty acid biomarkers were measured in plasma phospholipids ${ }^{(30,32,38,44)}$, plasma cholesterol esters ${ }^{(44)}$; red blood cell membranes ${ }^{(34)}$; red blood cell phospholipids ${ }^{(35,38)}$, by gas or gas-liquid chromatography.

$\ddagger$ Degree of covariate adjustment indicated by $(+)$ : sociodemographics; $(++)$ : sociodemographics and other risk factors; $(+++)$ : sociodemographics and other risk factors and dietary variables.

§Quality of each study was assessed by 5 separate criteria on an integer scale ( 0 or 1 , with 1 being better). These included: appropriateness and reporting of inclusion and exclusion criteria, assessment of exposure (1 point if habitual $n-3$ PUFA/fish/seafood consumption was assessed with a validated diet assessment method, i.e. a validated FFQ or repeated short term measures; for biomarkers, a published laboratory assay), assessment of outcome ( 1 point if diagnosis of DM was confirmed according to accepted criteria and not based on self report), control of confounding (1 point if adjusted for socio-demographic plus either other risk factors or dietary variables for dietary studies; 1 point if adjusted for socio-demographic plus other risk factors for biomarker studies) and evidence of bias (1 point if no evidence of bias). Scores were summed and studies with scores from 0 to 3 and 4 to 5 were considered lower and higher quality, respectively.

|| Dependent on the study, additional data requested included baseline characteristics (age, BMI, exposure distributions) and exposure category-specific data (number of participants, person-years of follow-up, number of cases, median level of exposure, risk estimates and $95 \% \mathrm{Cl}$.

I Nested selection of cases and controls within the original cohort. The total number of participants recruited in the original cohorts were: Melbourne Collaborative Cohort Study, $n 41528^{(32)}$; Vasterbotton intervention program ${ }^{(34)}$, $n$ 33 336; EPIC-Norfolk, $n 25639^{(38)}$

** Dietary EPA+DHA in these studies were imputed from fish/seafood intake by using a conversion factor calculated from nationally representative dietary surveys as part of our work in the Global Burden of Diseases study ${ }^{(48)}$

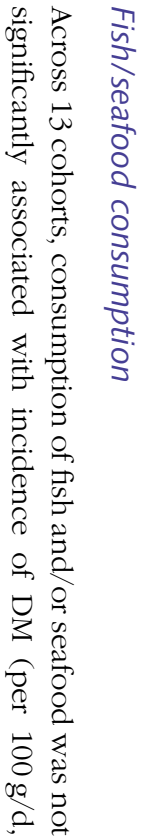

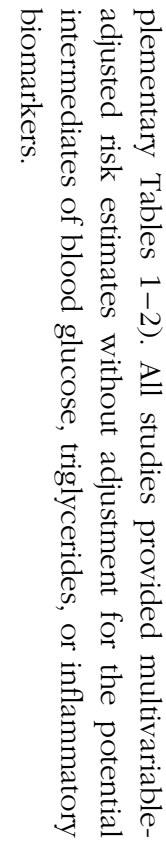

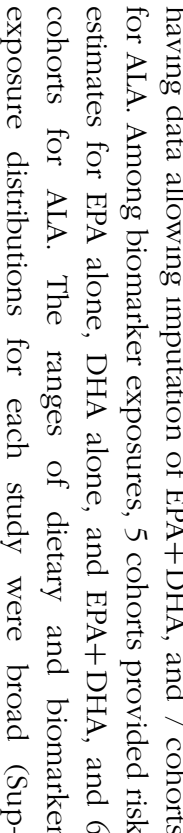

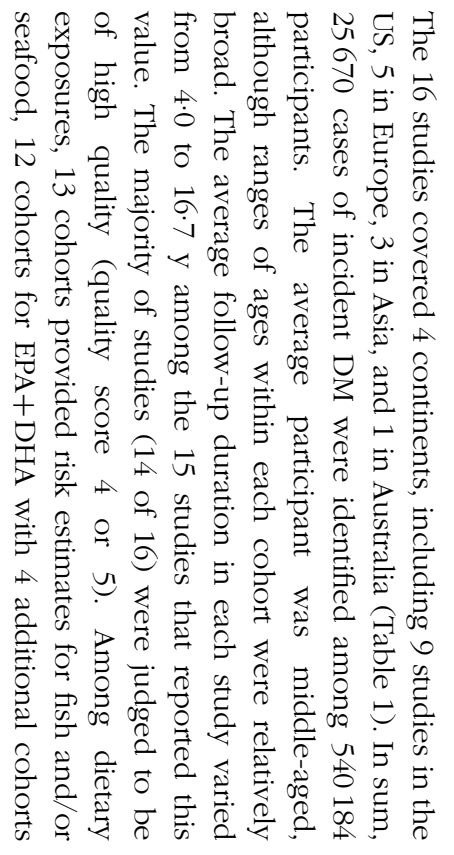

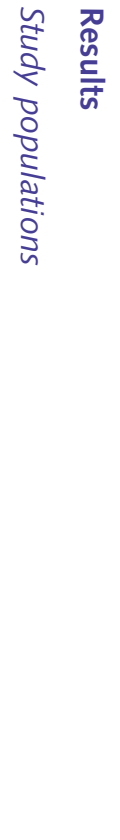

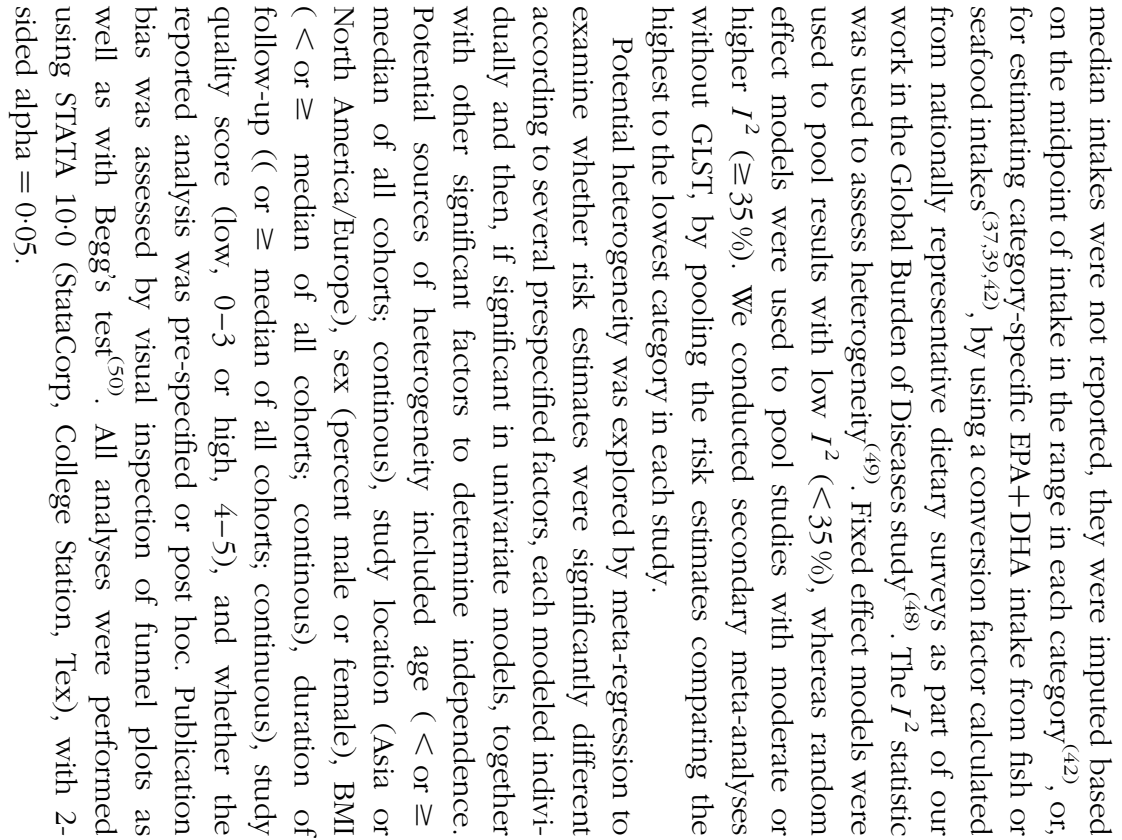




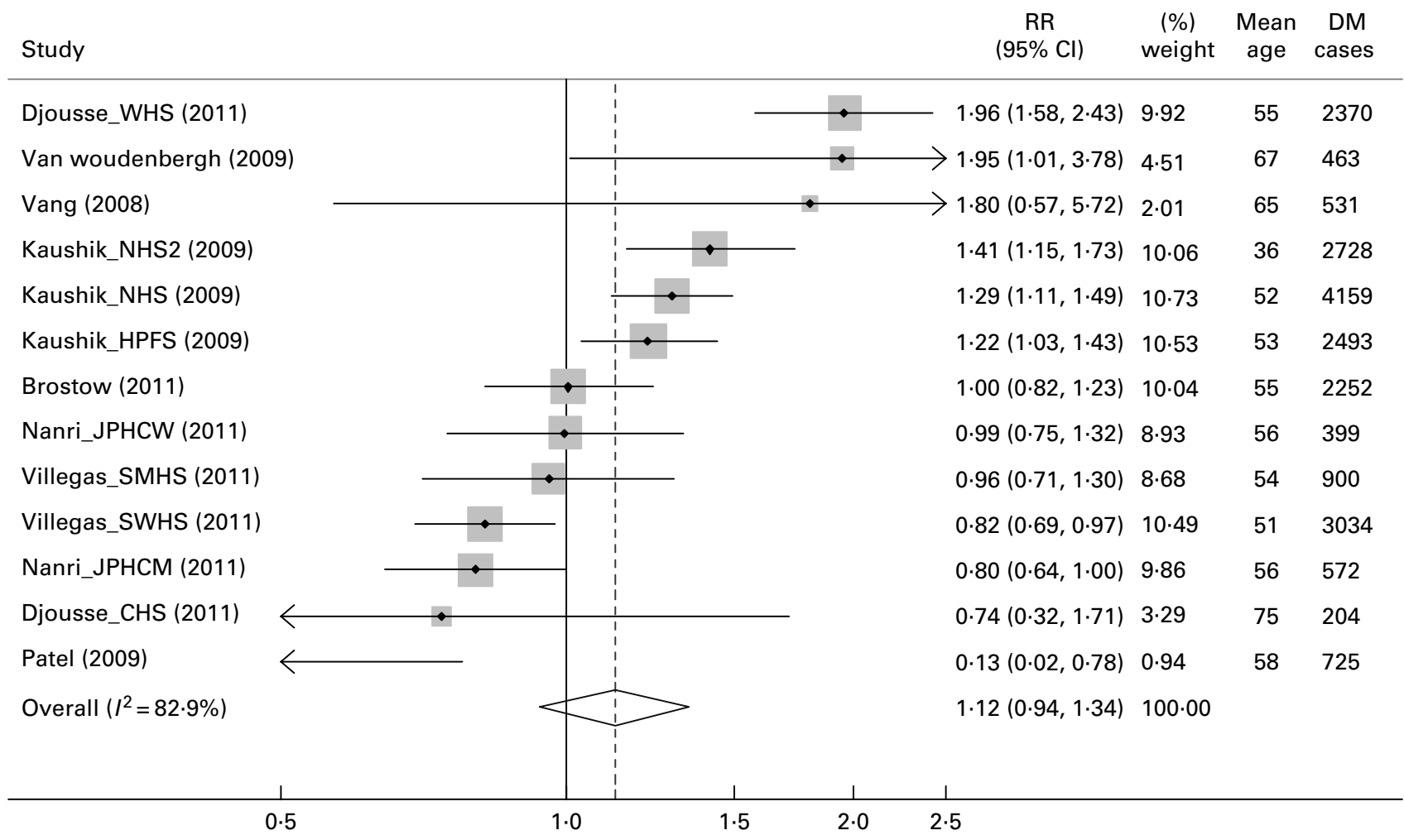

Fig. 2. Relative risk of type 2 diabetes associated with fish and/or seafood consumption in 13 prospective cohorts including 481489 participants and 20830 cases of incident diabetes. Within-study relative risks and $95 \%$ Cl's were quantified using generalized least squares trend estimation, and study-specific results were pooled using random effect meta-analysis. For 3 cohorts reporting effect estimates for both fish and seafood intake, ${ }^{(29,43)}$ effects estimates for fish were used in the primary analysis; findings using seafood did not appreciably alter results (not shown).

$\mathrm{RR}=1 \cdot 12,95 \% \mathrm{CI}=0 \cdot 94,1 \cdot 34, \mathrm{P}=0 \cdot 21$, Fig. 2). Substantial between-study heterogeneity was evident $\left(I^{2}=82 \cdot 9 \%\right)$. In stratified analyses and unadjusted meta-regressions, study location (Asia vs. North America/Europe), mean BMI, and duration of follow-up each significantly modified the association of fish/seafood intake and risk of DM. Lower risk was seen in studies from Asia, in studies with lower mean BMI $\left(<24.5 \mathrm{~kg} / \mathrm{m}^{2}\right)$, and in studies with shorter durations of follow-up $(<10 \mathrm{y})$; and higher risk in studies from North America/Europe, with higher mean BMI $\left(\geq 24.5 \mathrm{~kg} / \mathrm{m}^{2}\right)$, and with longer durations of follow-up ( $\geq 10 \mathrm{y}$ ) (P-Interaction $\leq 0.007$ each). For example, fish/seafood was associated with lower DM risk in Asia cohorts (RR per $100 \mathrm{~g} / \mathrm{d}=0.89$, $95 \% \mathrm{CI}=0.81,0.98)$, and higher risk in North America/ Europe cohorts (RR per $100 \mathrm{~g} / \mathrm{d}=1 \cdot 38,95 \% \mathrm{CI}=1 \cdot 13,1 \cdot 70$ ) (Fig. 3).

Because study location, mean BMI, and duration of followup were highly collinear across studies - the cohorts from Asia were generally the same as those with lower mean BMI and shorter durations of follow-up (Fig. 3) - none of these three factors remained statistically predictive of heterogeneity in multivariable meta-regression including all three factors $(P \geq 0 \cdot 18$ each). Even among studies restricted to North America/Europe, substantial unexplained heterogeneity remained $\left(I^{2}=70 \cdot 1 \%\right)$; no heterogeneity was evident across studies in Asia $\left(I^{2}=0 \%\right)$.

When we repeated meta-analysis of risk estimates comparing the top to the bottom exposure category in each study, similar results were seen, with no overall pooled association between fish/seafood consumption and incidence of diabetes $\left(\mathrm{RR}=1.07, \quad 95 \% \quad \mathrm{CI}=0.94, \quad 1 \cdot 22, \quad P=0.29, \quad I^{2}=77.7 \%\right)$. When evaluated separately, study location, mean BMI, and duration of follow-up each again significantly modified the association between fish/seafood intake and risk of DM ( $P$-interaction $<0.05$ each), with similar collinearity as above. Overall findings were also similar when restricted to the 8 risk estimates for fish consumption alone or the 8 risk estimates for seafood consumption alone (data not shown).

\section{Dietary EPA and DHA}

Across 16 cohorts, estimated EPA+DHA consumption was not associated with DM risk (per $250 \mathrm{mg} / \mathrm{d}, \quad \mathrm{RR}=1 \cdot 04,95 \%$ $\mathrm{CI}=0 \cdot 97-1 \cdot 10, \quad P=0 \cdot 27$, Fig. 4). Substantial heterogeneity was evident $\left(I^{2}=82 \%\right)$. Because these studies of estimated $\mathrm{EPA}+\mathrm{DHA}$ consumption were largely overlapping with the studies of fish consumption described above, in unadjusted meta-regressions this heterogeneity again appeared partly related to varying study location, mean BMI, and durations of follow-up ( $P$-interaction $\leq 0.02$ each), with similar collinearity among these factors as described previously. For example, in studies in Asia, EPA+DHA consumption was associated with lower incidence of DM (per $250 \mathrm{mg} / \mathrm{d}$, RR $=0.95,95 \%$ $\mathrm{CI}=0.91,0.99)$, with minimal heterogeneity across studies $\left(I^{2}=14.7 \%\right)$; whereas in studies in North America/Europe, $\mathrm{EPA}+\mathrm{DHA}$ consumption was associated with higher incidence 


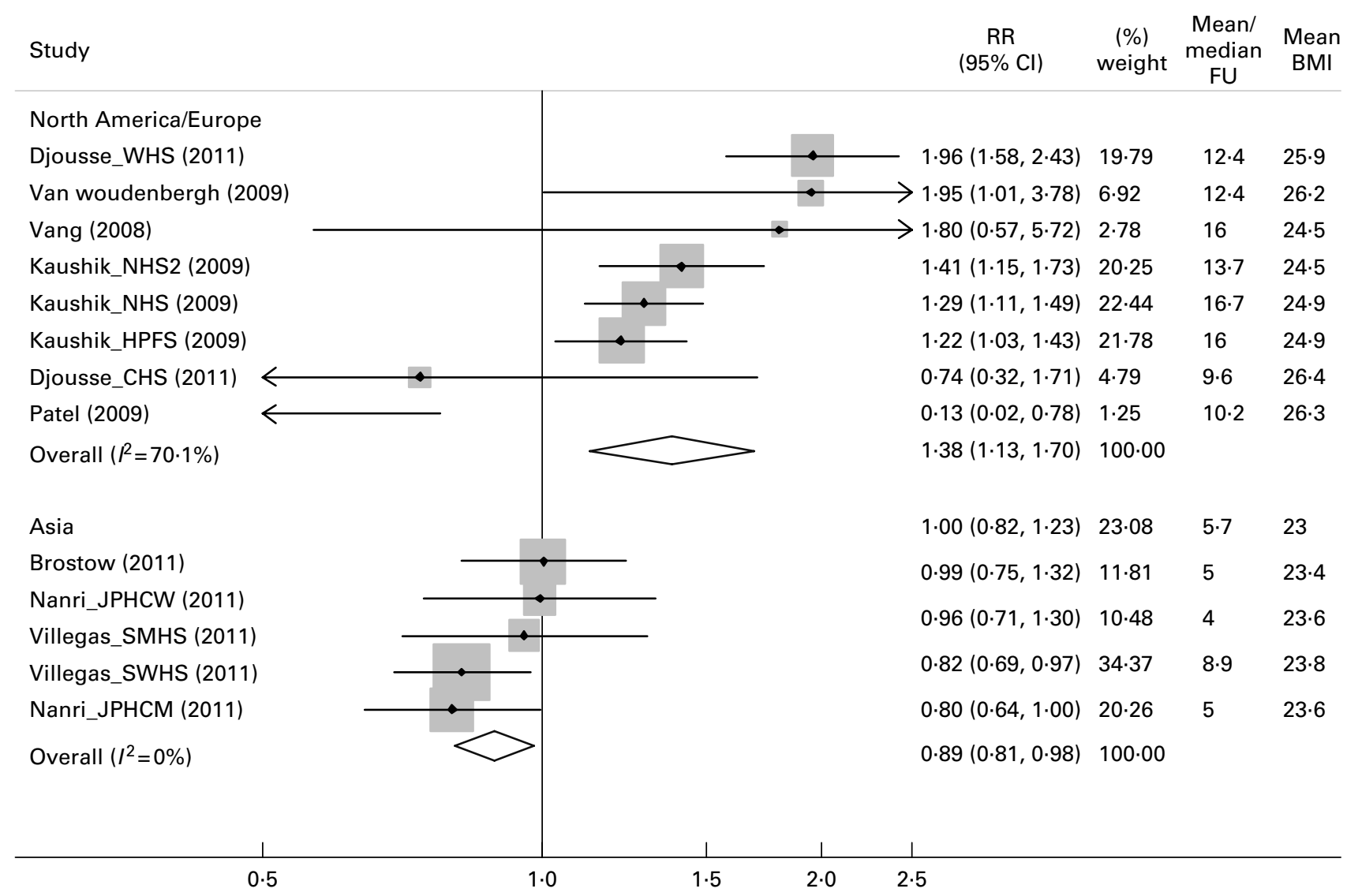

Fig. 3. Relative risk of type 2 diabetes associated with fish and/or seafood consumption in prospective cohort studies stratified according to study location in North America/Europe (top panel) vs. Asia (bottom panel). Within-study relative risks and $95 \%$ Cl's were quantified using generalized least squares trend estimation, and study-specific results were pooled using random effect meta-analysis. Effect modification by study location was significant $(P$-Interaction=0.007).

of $\mathrm{DM}($ per $250 \mathrm{mg} / \mathrm{d}, \mathrm{RR}=1 \cdot 12,95 \% \mathrm{CI}=1 \cdot 05,1 \cdot 20)$, with substantial remaining heterogeneity $\left(I^{2}=67.5 \%\right) \quad$ (Supplementary Fig. 1).

Meta-analysis of risk estimates comparing the top vs. bottom exposure category in each study gave similar results, with no overall pooled association between EPA+DHA consumption and incidence of diabetes $(\mathrm{RR}=1 \cdot 04,95 \% \mathrm{CI}=0.94,1 \cdot 16$, $\left.P=0 \cdot 46, I^{2}=79 \cdot 0 \%\right)$. Among the 5 cohorts reporting risk estimates for estimated dietary EPA or DHA separately, neither EPA $($ per $125 \mathrm{mg} / \mathrm{d}, \mathrm{RR}=1.07,95 \% \mathrm{CI}=0.85,1.34, P=0.58)$ nor DHA (per $125 \mathrm{mg} / \mathrm{d}, \mathrm{RR}=1 \cdot 04,95 \% \mathrm{CI}=0.90,1 \cdot 21$, $P=0.59)$ were significantly associated with incidence of $\mathrm{DM}$, although substantial heterogeneity was present for both estimates $\left(I^{2}=77 \cdot 4 \%\right.$ and $80 \cdot 2 \%$, respectively).

\section{Circulating $E P A+D H A$}

Among 5 cohorts that evaluated circulating EPA+DHA biomarkers, no association was seen between EPA+DHA concentrations and incidence of DM: per 3\% of total fatty acids, $\mathrm{RR}=0.94,95 \% \mathrm{CI}=0.75,1 \cdot 17, P=0.56$ (Fig. 5). Moderate heterogeneity was evident $\left(I^{2}=40 \cdot 4 \%\right)$. Meta-regression did not identify any statistically significant sources of heterogeneity, although statistical power was limited due to only 5 studies. Four cohorts were from North America/Europe, and one from Australia; none were from Asia. Excluding the Australian cohort, EPA+DHA concentrations were not associated with higher incidence of DM: per $3 \%$ of total fatty acids, $\mathrm{RR}=0.88,95 \% \mathrm{CI}=0.72,1 \cdot 08)$. Meta-analysis of risk estimates for the top vs. bottom exposure category in each study gave similar results $(\mathrm{RR}=0.94,95 \% \mathrm{CI}=0.82,1 \cdot 09$, $I^{2}=47 \%$ ). When circulating EPA and DHA were evaluated separately, neither EPA (per $1 \%$ of total fatty acids, $\left.\mathrm{RR}=0.96,95 \% \mathrm{CI}=0 \cdot 86,1 \cdot 07, P=0 \cdot 48, I^{2}=0 \%\right)$ nor DHA (per $1 \%$ of total fatty acids, $\mathrm{RR}=1 \cdot 00,95 \% \mathrm{CI}=0 \cdot 91,1 \cdot 10$, $P=0 \cdot 97, I^{2}=0 \%$ ) were significantly associated with incidence of DM.

\section{Dietary ALA}

Across 7 cohort studies, estimated ALA consumption was associated with a non-significant trend toward lower incidence of DM (per 0.5 g/day, RR $=0.93,95 \% \mathrm{CI}=0.83,1.04$, $P=0 \cdot 20$, Fig. 6). Heterogeneity was present $\left(I^{2}=54 \cdot 3 \%\right)$, but no statistically significant sources of heterogeneity were identified by meta-regression, although power was limited. Similar results were obtained in meta-analysis of risk estimates comparing the top to the bottom exposure category in each study $\left(\mathrm{RR}=0 \cdot 92,95 \% \mathrm{CI}=0 \cdot 80,1 \cdot 05, P=0 \cdot 21, I^{2}=40 \cdot 3 \%\right)$. 
Study

RR

(\%) Mean DM

$(95 \% \mathrm{Cl})$

Weight Age cases

Vang (2008)

Djousse_WHS (2011)

Kaushik_NHS2 (2009)

Kroger (2011)

Van woudenbergh (2009)

Kaushik_NHS (2009)

Meyer (2001)

Kaushik_HPFS (2009)

Nanri_JPHCW (2011)

Djousse_CHS (2011)

Nanri_JPHCM (2011)

Brostow (2011)

Villegas_SMHS (2011)

Hodge (2007)

Villegas_SWHS (2011)

Patel (2009)

Overall $(R=82 \cdot 0 \%)$

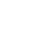

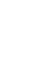

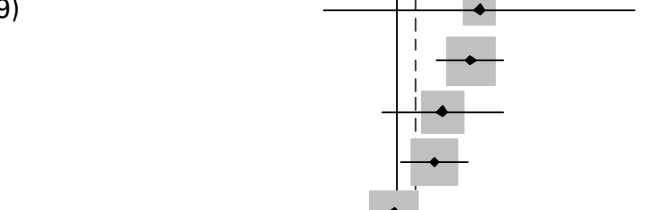

$>1.45(0.70,2.97) 0.72$

$1.30(1.19,1.42) \quad 8.12 \quad 55 \quad 2370$

$1.20(1.09,1.32) \quad 7.94 \quad 36 \quad 2728$

$1.16(1.01,1.32) 6.79 \quad 51 \quad 673$

$1.15(0.89,1.49) \quad 3.68 \quad 67 \quad 463$

$1.13(1.07,1.20) \quad 8.97 \quad 52 \quad 4159$

$1.08(0.98,1.20) 7.66 \quad 62 \quad 1890$

$1.07(1.01,1.13) \quad 8.91 \quad 53 \quad 2493$

$1.00(0.93,1.07) 8.63 \quad 56 \quad 399$

$0.98(0.83,1.16) \quad 5.89 \quad 75 \quad 204$

$0.95(0.90,1.00) 9.01 \quad 56 \quad 572$

$0.95(0.86,1.05) \quad 7.75 \quad 55 \quad 2252$

$0.90(0.67,1.21) \quad 3.15 \quad 54 \quad 900$

$0.85(0.69,1.06) \quad 4.63 \quad 55 \quad 346$

$0.83(0.71,0.97) \quad 6.03 \quad 51 \quad 3034$

$0.64(0.44,0.95) \quad 2.11 \quad 58 \quad 725$

$1.04(0.97,1.10) \quad 100.00$

\section{Circulating ALA}

In 6 cohorts that assessed circulating ALA biomarkers, higher ALA levels were associated with a non-significant trend towards lower risk of DM (per $0 \cdot 1 \%$ of total fatty acids, $\mathrm{RR}=0 \cdot 90,95 \% \mathrm{CI}=0 \cdot 80,1 \cdot 00, P=0 \cdot 06$, Fig. 7). Betweenstudy heterogeneity was not evident $\left(I^{2}=17.9 \%\right)$. Results were similar in meta-analysis of risk estimates in the top vs. bottom exposure category in each study $(\mathrm{RR}=0 \cdot 87,95 \%$ $\left.\mathrm{CI}=0 \cdot 74,1 \cdot 01, P=0 \cdot 07, I^{2}=18 \cdot 9 \%\right)$.

\section{Publication bias}

For dietary fish/seafood intake, visual inspection of the funnel plot found that individual study RR estimates were reasonably symmetrical about the pooled effect estimate, suggesting no evidence of publication bias (Supplementary Fig. 2, top panel). This was supported by a null Begg's test for publication bias $(P=0.45)$. For dietary EPA+DHA intake, the funnel plot showed slightly more data points among the smaller studies to the left of the pooled estimate of RR, indicating possible publication bias in favor of a protective association (Supplementary Fig. 2, bottom panel). Begg's test $(P=0.59)$ suggested no significant evidence of publication bias. There was also little evidence for publication bias for all other exposure-outcome relationships based on either visual inspection of funnel plots or Begg's test (data not shown).

\section{Discussion}

The overall findings from this systematic review and metaanalysis suggest that dietary EPA+DHA and fish/seafood consumption do not have either major harmful or beneficial associations with the development of DM. However, we identified substantial heterogeneity in findings across studies. Our findings also suggest that plant-derived ALA could be protective, though this result was of borderline statistical significance.

\section{Fish/seafood intake, n-3 PUFA, and DM risk}

We found little evidence that fish or seafood, dietary $\mathrm{EPA}+\mathrm{DHA}$, or circulating EPA+DHA biomarkers were associated with risk of DM overall. These studies typically comprised moderately overweight but otherwise generally healthy participants at baseline. Prior short-term randomized controlled trials have found very little effect of $n$-3 PUFA supplementation on glucose metabolism or indices of insulin resistance in healthy subjects ${ }^{(5,51-53)}$. Therefore, our overall findings are consistent with these prior metabolic trials and suggest 


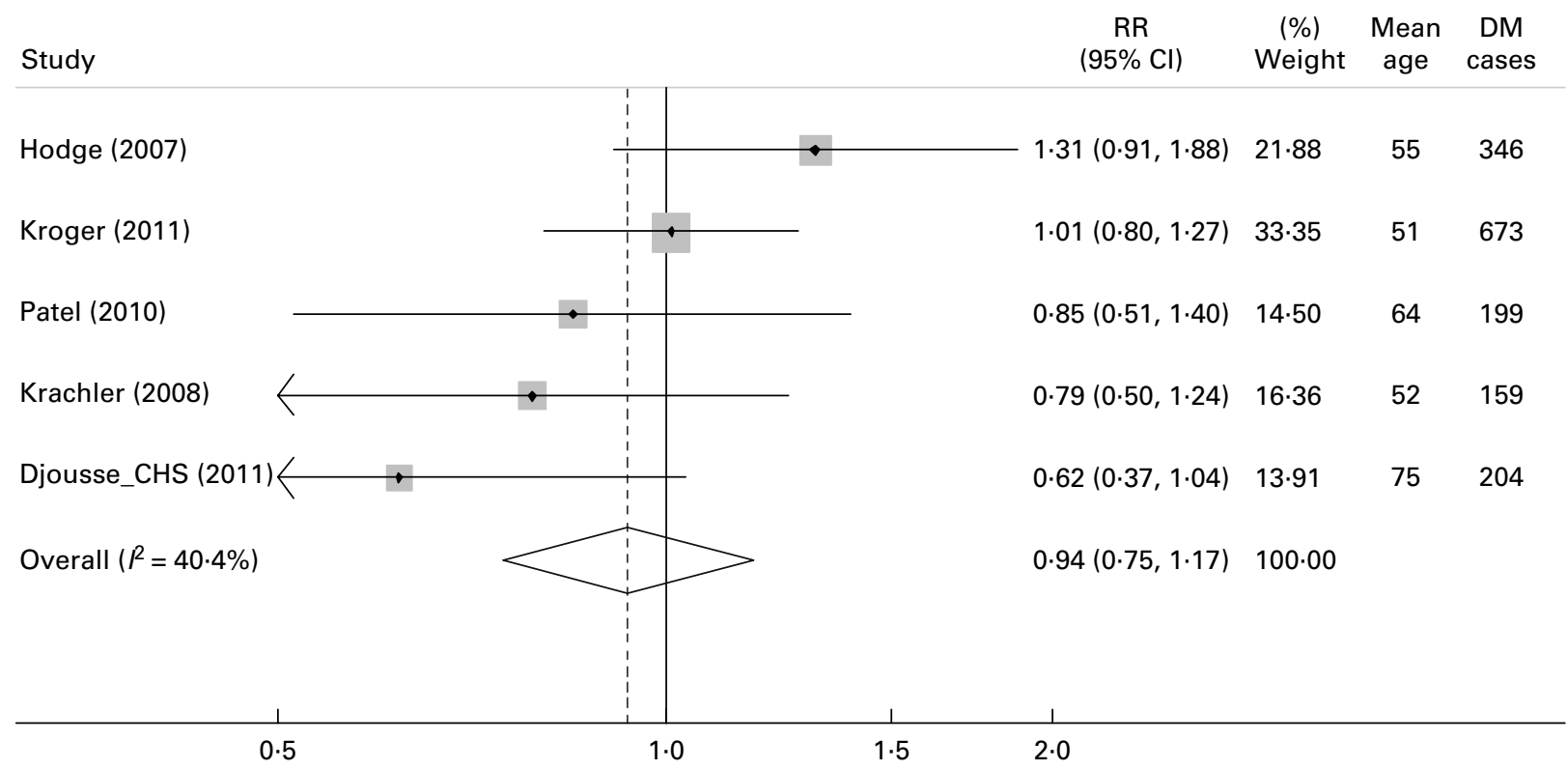

Fig. 5. Relative risk of type 2 diabetes associated with EPA+DHA biomarker concentrations (as \% of total fatty acids) in 5 prospective cohorts including 10382 individuals and 1581 incident diabetes cases. Within-study relative risks and $95 \%$ Cl's were quantified using generalized least squares trend estimation, and study-specific results were pooled using random effect meta-analysis. Pooling results using the alternative biomarker measurements (red blood cell phospholipids) in Patel et al ${ }^{(38)}$, did not appreciably alter the results (per $3 \%$ of total fatty acids, $R R=0.96,95 \% \mathrm{Cl}=0.76-1.21$ ). The study by Wang et al ${ }^{(44)}$, was not included due to insufficient information to allow extraction of effect estimates. However, EPA and DHA were not associated with risk of diabetes ${ }^{(44)}$, and inclusion of this study is unlikely to appreciably alter the observed results.

that, at typical dietary levels of consumption in generally healthy subjects, fish, seafood, or dietary EPA+DHA may have minimal effects on development of DM.

Our results also demonstrate large heterogeneity between study results. Such heterogeneity could be due to chance, or related to variations in population characteristics, geographical locations, or study methodology. Studies included in the metaanalyses generally had high quality score $(\geq 4)$ and were prespecified analyses, and these factors were not significant sources of heterogeneity. Conversely, EPA+DHA and fish/seafood consumption were associated with lower risk of DM in studies conducted in Asia, and higher risk of DM among

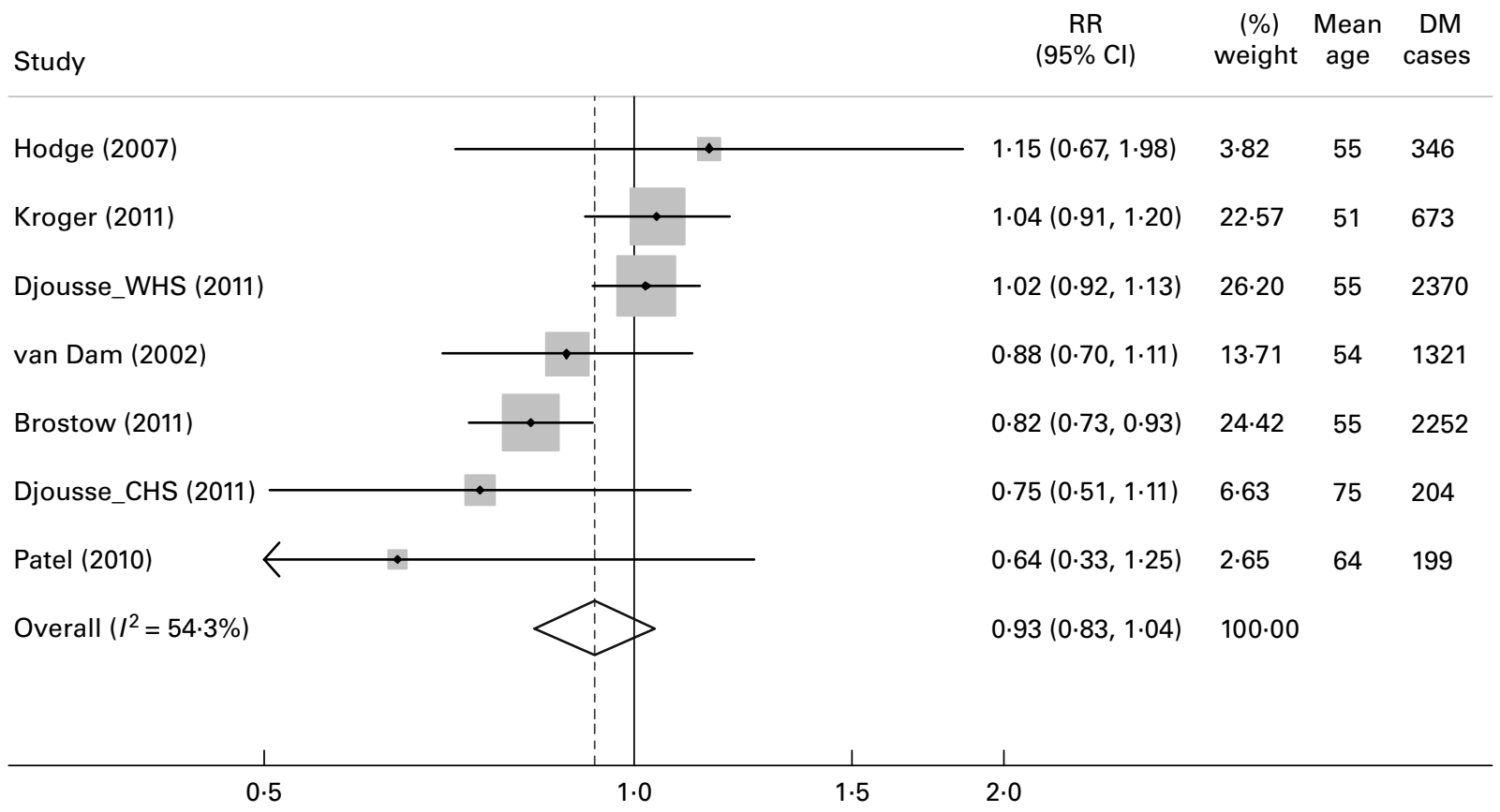

Fig. 6. Relative risk of type 2 diabetes associated with dietary ALA in 7 prospective cohorts including 131940 individuals and 7365 incident DM cases. Withinstudy relative risks and $95 \%$ Cl's were quantified using generalized least squares trend estimation, and study-specific results were pooled using random effect meta-analysis. 


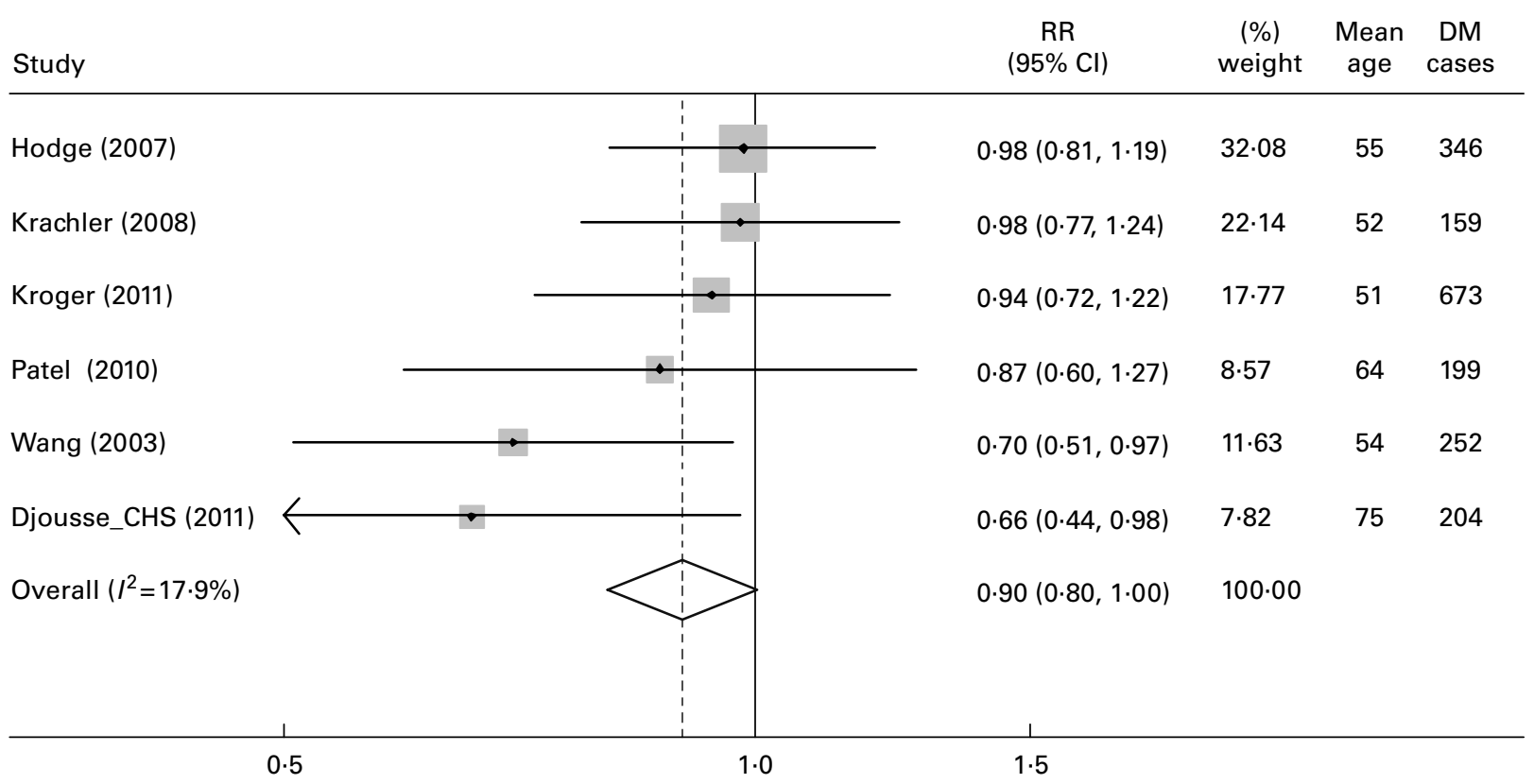

Fig. 7. Relative risk of type 2 diabetes associated with ALA biomarker (as \% of total fatty acids) in 6 prospective cohorts including 13291 individuals and 1833 incident diabetes cases. Within-study relative risks and $95 \%$ Cl's were quantified using generalized least squares trend estimation, and study-specific results were pooled using random effect meta-analysis. Pooling results using the alternative lipid compartment of biomarker measurements from Wang et al. ${ }^{(44)}$ and Patel et al. ${ }^{(38)}$ did not appreciably alter the results (per $0.1 \%$ of total fatty acids, $\mathrm{RR}=0.93,95 \% \mathrm{Cl}=0.85-1.02, P=0.11$ ).

studies conducted in North America/Europe. Mean cohort BMI and duration of follow-up were also potential sources of heterogeneity, but these study characteristics tracked with study location and their independent effects could not be confirmed.

Reasons for heterogeneity of effects by region could include true biologic diversity, publication bias, or chance. For example, average types of fish consumed or fish preparation methods may be different in Asia vs. North America/Europe, with potentially greater fatty fish and raw/steamed fish in the former, vs. white fish or deep fried fish in the latter, that could influence risk of DM. Pathophysiologic pathways for DM risk could also differ, and be differently influenced by $n$-3 fatty acids, in Asian vs. Caucasian populations; for example, Asians suffer DM at much lower BMI, with reasons not yet fully understood ${ }^{(54,55)}$. Given potential benefits of fish or EPA+DHA consumption on total mortality, ${ }^{(56)}$ greater survivorship could also be a partial explanation for higher risk of DM in longer-term studies, but this seems less likely to us and should be evaluated by assessment of competing mortality in future studies. All of these subgroup findings may also be due to chance and also should be interpreted cautiously as all analyses of heterogeneity were based on average study-level, rather than individual-level, characteristics. Additional studies in diverse cultures and geographical regions, as well as studies assessing potential interaction with BMI or individual-level duration of follow-up, should explore these issues. Notably, studies that used objective biomarkers of EPA+DHA found no evidence for higher diabetes risk, including cohorts from North America/ Europe. This suggests that the observed higher risk in stratified analyses including only North America/Europe studies that utilized self-reported estimates of fish or EPA+DHA consumption could be partly related to the dietary assessment method, rather than to biologic effects of EPA+DHA per se.

\section{$A L A$ and $D M$ risk}

Compared with seafood sources, we identified fewer prospective studies of ALA and incident DM. We found that both estimated dietary consumption and circulating biomarkers of ALA were associated with a modest, nonstatistically significant trend towards lower risk of DM. These pooled estimates demonstrated relatively low heterogeneity between-studies, suggesting more consistent findings among studies. In several animal models, dietary ALA or flaxseed oil (a rich source of ALA) improved insulin sensitivity and glycemic responses ${ }^{(57-59)}$. Similarly, some ${ }^{(60,61)}$, but not other ${ }^{(53)}$, short-term randomized clinical trials found that ALA or flaxseed oil moderately improved fasting plasma glucose and markers of insulin resistance in humans. Our current results add to these limited but potentially important data that ALA may provide moderate protection against the development of DM. Because plant sources of $n$-3 PUFA are potentially more widely available on a global basis, our findings highlight the need for further clinical and observational investigation of these effects.

\section{Strength and limitations}

Our analysis had several strengths. Multiple databases were systematically searched to ensure identification of all relevant published studies, and we also obtained clarification and/or additional data from several authors to minimize misclassification and potential for publication bias. The prospective 
cohort design of the included studies reduces the possibility of recall and selection bias. The large number of total incident DM cases provided statistical power to detect clinically meaningful associations. We pooled all available information from each study using GLST, which accounts for both categories of exposure and dose-response, rather than simply pooling often incomparable single extreme categories. We evaluated both dietary estimates and objective biomarkers of $n-3$ PUFA, and consistent findings for each increased confidence in validity of our findings. Studies were identified from wide geographical locations (North America, Europe, Asia, Australia) with varied population characteristics (age, BMI, sex, exposure distributions), increasing generalizability.

Potential limitations should be considered. Although most studies adjusted for major sociodemographic, lifestyle, clinical, and other dietary risk factors, the possibility of residual confounding by unmeasured or imprecisely measured factors remains a possibility, particularly as both seafood and ALA consumption are often associated with healthier lifestyles. Conversely, measurement error in exposures could have attenuated true effects towards the null, causing underestimation of associations. The consistency of findings for both dietary and biomarker exposures is reassuring, as self-reported dietary estimates and $n-3$ PUFA biomarkers are subject to differing types of error and sources of confounding. Most studies evaluated total fish and/or seafood consumption, and we were unable to evaluate specific fish species or preparation methods which might influence effects on DM. As in all meta-analyses, publication bias is possible. However, visual inspection of funnel plots did not suggest publication bias was a substantial problem; our direct contact with authors and experts minimized the possibility of missing unpublished studies; and the overall null pooled findings are typically less subject to influence from publication bias than significant positive or inverse relations.

\section{Conclusions}

Based on all available evidence from prospective studies, neither EPA+DHA nor fish/seafood intake have significant associations with risk of DM overall, while plant-derived ALA is associated with nonsignificant trends toward lower risk.

\section{Acknowledgements}

The study was supported by the NHLBI, NIH, with additional support from the Office of Dietary Supplements (R01 HL085710) and by a Research Fellowship from the National Heart Foundation of Australia. We thank the following authors for providing clarification on their published articles and/or additional unpublished data: Aaron Folsom, Katie Meyer, Lu Wang, Allison Hodge, Benno Krachler, Nita Forouhi, Pinal Patel, Geertuida van Woudenbergh, Andrew Odegaard, Janine Kröger, Akiko Nanri, and Raquel Villegas. We would also like to thank the NHLBI, NIH and National Heart Foundation of Australia for funding support. Conflict of Interest Statement: Dr. Mozaffarian reports research grants from GlaxoSmithKline, Sigma Tau, Pronova (significant), ad hoc travel reimbursement and/or honoraria from International Life Sciences Institute, Unilever, Bunge, SPRIM, Nutrition Impact (modest); ad hoc consulting fees from Foodminds, McKinsey Health Systems Institute (modest); and royalties from UpToDate (modest). The other authors report no conflicts of interest. Author Contributions: Jason Wu: study concept and design; data extraction and statistical analysis; interpretation of data; drafting of the manuscript; critical revision of the manuscript for important intellectual content; approval of final manuscript for submission. Renata Micha: data extraction; interpretation of data; critical revision of the manuscript for important intellectual content; approval of final manuscript for submission. Fumiaki Imamura: statistical advice; interpretation of data; critical revision of the manuscript for important intellectual content; approval of final manuscript for submission. An Pan: interpretation of data; critical revision of the manuscript for important intellectual content; approval of final manuscript for submission. Mary Biggs: interpretation of data; critical revision of the manuscript for important intellectual content; approval of final manuscript for submission. Owais Ajaz: interpretation of data; critical revision of the manuscript for important intellectual content; approval of final manuscript for submission. Luc Djousse: interpretation of data; critical revision of the manuscript for important intellectual content; approval of final manuscript for submission. Frank Hu: interpretation of data; critical revision of the manuscript for important intellectual content; approval of final manuscript for submission. Dariush Mozaffarian: study concept and design; statistical advice; obtained funding; drafting the manuscript; interpretation of data; critical revision of the manuscript for important intellectual content; approval of final manuscript for submission. Supported by the NHLBI, NIH, with additional support from the Office of Dietary Supplements (R01 HL085710) and by a Research Fellowship from the National Heart Foundation of Australia. The supporting agencies had no role in the design or conduct of the study, the collection, management, analysis, or interpretation of the data, or the preparation, review, and approval of the manuscript.

\section{References}

1. Steyn NP, Mann J, Bennett PH, et al. (2004) Diet, nutrition and the prevention of type 2 diabetes. Public Health Nutr 7, 147-165.

2. Schulze MB \& Hu FB (2005) Primary prevention of diabetes: what can be done and how much can be prevented? Annu Rev Public Health 26, 445-467.

3. Banga A, Unal R, Tripathi P, et al. (2009) Adiponectin translation is increased by the PPARgamma agonists pioglitazone and omega-3 fatty acids. Am J Physiol Endocrinol Metab 296, E480-E489.

4. Vemuri M, Kelley DS, Mackey BE, et al. (2007) Docosahexaenoic Acid (DHA) But Not Eicosapentaenoic Acid (EPA) Prevents Trans-10, Cis-12 Conjugated Linoleic Acid (CLA)Induced Insulin Resistance in Mice. Metab Syndr Relat Disord 5, 315-322.

5. Itoh M, Suganami T, Satoh N, et al. (2007) Increased adiponectin secretion by highly purified eicosapentaenoic acid in 
rodent models of obesity and human obese subjects. Arterioscler Thromb Vasc Biol 27, 1918-1925.

6. Perez-Matute P, Perez-Echarri N, Martinez JA, et al. (2007) Eicosapentaenoic acid actions on adiposity and insulin resistance in control and high-fat-fed rats: role of apoptosis, adiponectin and tumour necrosis factor-alpha. $\mathrm{Br} J \mathrm{Nutr}$ 97, 389-398.

7. Oh DY, Talukdar S, Bae EJ, et al. (2010) GPR120 is an omega-3 fatty acid receptor mediating potent anti-inflammatory and insulin-sensitizing effects. Cell 142, 687-698.

8. Gonzalez-Periz A, Horrillo R, Ferre N, et al. (2009) Obesityinduced insulin resistance and hepatic steatosis are alleviated by omega-3 fatty acids: a role for resolvins and protectins. Faseb J 23, 1946-1957.

9. Serhan CN, Chiang N \& Van Dyke TE (2008) Resolving inflammation: dual anti-inflammatory and pro-resolution lipid mediators. Nat Rev Immunol 8, 349-361.

10. Tanaka N, Zhang X, Sugiyama E, et al. (2010) Eicosapentaenoic acid improves hepatic steatosis independent of PPARalpha activation through inhibition of SREBP-1 maturation in mice. Biochem Pharmacol 80, 1601-1612.

11. Neschen S, Morino K, Dong J, et al. (2007) n-3 Fatty acids preserve insulin sensitivity in vivo in a peroxisome proliferator-activated receptor-alpha-dependent manner. Diabetes 56, 1034-1041.

12. Sato A, Kawano H, Notsu T, et al. (2010) Antiobesity effect of eicosapentaenoic acid in high-fat/high-sucrose diet-induced obesity: importance of hepatic lipogenesis. Diabetes 59 , 2495-2504.

13. Jump DB (2011) Fatty acid regulation of hepatic lipid metabolism. Curr Opin Clin Nutr Metab Care 14, 115-120.

14. Friedberg CE, Janssen MJ, Heine RJ, et al. (1998) Fish oil and glycemic control in diabetes. A meta-analysis. Diabetes Care 21, 494-500.

15. Hartweg J, Perera R, Montori V, et al. (2008) Omega-3 polyunsaturated fatty acids (PUFA) for type 2 diabetes mellitus. Cochrane Database Syst Rev, CD003205.

16. Montori VM, Farmer A, Wollan PC, et al. (2000) Fish oil supplementation in type 2 diabetes: a quantitative systematic review. Diabetes Care 23, 1407-1415.

17. Fedor D \& Kelley DS (2009) Prevention of insulin resistance by $n-3$ polyunsaturated fatty acids. Curr Opin Clin Nutr Metab Care 12, 138-146.

18. Stroup DF, Berlin JA, Morton SC, et al. (2000) Meta-analysis of observational studies in epidemiology: a proposal for reporting. Meta-analysis Of Observational Studies in Epidemiology (MOOSE) group. JAMA 283, 2008-2012.

19. Salmeron J, Hu FB, Manson JE, et al. (2001) Dietary fat intake and risk of type 2 diabetes in women. Am J Clin Nutr 73, 1019-1026.

20. Schulze MB, Manson JE, Willett WC, et al. (2003) Processed meat intake and incidence of Type 2 diabetes in younger and middle-aged women. Diabetologia 46, 1465-1473.

21. Song Y, Manson JE, Buring JE, et al. (2004) A prospective study of red meat consumption and type 2 diabetes in middle-aged and elderly women: the women's health study. Diabetes Care 27, 2108-2115.

22. Krishnan S, Coogan PF, Boggs DA, et al. (2010) Consumption of restaurant foods and incidence of type 2 diabetes in African American women. Am J Clin Nutr 91, 465-471.

23. Feskens EJ, Virtanen SM, Rasanen L, et al. (1995) Dietary factors determining diabetes and impaired glucose tolerance. A 20-year follow-up of the Finnish and Dutch cohorts of the Seven Countries Study. Diabetes Care 18, 1104-1112.
24. Vessby B, Aro A, Skarfors E, et al. (1994) The risk to develop NIDDM is related to the fatty acid composition of the serum cholesterol esters. Diabetes 43, 1353-1357.

25. Colditz GA, Manson JE, Stampfer MJ, et al. (1992) Diet and risk of clinical diabetes in women. Am J Clin Nutr $\mathbf{5 5}$, 1018-1023.

26. Harding AH, Day NE, Khaw KT, et al. (2004) Dietary fat and the risk of clinical type 2 diabetes: the European prospective investigation of Cancer-Norfolk study. Am J Epidemiol 159, $73-82$

27. Feskens EJ, Bowles CH \& Kromhout D (1991) Inverse association between fish intake and risk of glucose intolerance in normoglycemic elderly men and women. Diabetes Care $\mathbf{1 4}$, 935-941.

28. Laaksonen DE, Lakka TA, Lakka HM, et al. (2002) Serum fatty acid composition predicts development of impaired fasting glycaemia and diabetes in middle-aged men. Diabet Med 19, 456-464.

29. Brostow DP, Odegaard AO, Koh WP, et al. (2011) Omega-3 fatty acids and incident type 2 diabetes: the Singapore Chinese Health Study. Am J Clin Nutr, In press.

30. Djousse L, Biggs ML, Lemaitre RN, et al. (2011) Plasma omega-3 fatty acids and incident diabetes in older adults. Am J Clin Nutr 94, 527-533.

31. Djousse L, Gaziano JM, Buring JE, et al. (2011) Dietary omega-3 fatty acids and fish consumption and risk of type 2 diabetes. Am J Clin Nutr 93, 143-150.

32. Hodge AM, English DR, O'Dea K, et al. (2007) Plasma phospholipid and dietary fatty acids as predictors of type 2 diabetes: interpreting the role of linoleic acid. Am J Clin Nutr 86, 189-197.

33. Kaushik M, Mozaffarian D, Spiegelman D, et al. (2009) Longchain omega- 3 fatty acids, fish intake, and the risk of type 2 diabetes mellitus. Am J Clin Nutr 90, 613-620.

34. Krachler B, Norberg M, Eriksson JW, et al. (2008) Fatty acid profile of the erythrocyte membrane preceding development of Type 2 diabetes mellitus. Nutr Metab Cardiovasc Dis 18, 503-510.

35. Kroger J, Zietemann V, Enzenbach C, et al. (2011) Erythrocyte membrane phospholipid fatty acids, desaturase activity, and dietary fatty acids in relation to risk of type 2 diabetes in the European Prospective Investigation into Cancer and Nutrition (EPIC)-Potsdam Study. Am J Clin Nutr 93, $127-142$.

36. Meyer KA, Kushi LH, Jacobs DR Jr, et al. (2001) Dietary fat and incidence of type 2 diabetes in older Iowa women. Diabetes Care 24, 1528-1535.

37. Nanri A, Mizoue T, Noda M, et al. (2011) Fish intake and type 2 diabetes in Japanese men and women: the Japan Public Health Center-based Prospective Study. Am J Clin Nutr 94, 884-891.

38. Patel PS, Sharp SJ, Jansen E, et al. (2010) Fatty acids measured in plasma and erythrocyte-membrane phospholipids and derived by food-frequency questionnaire and the risk of new-onset type 2 diabetes: a pilot study in the European Prospective Investigation into Cancer and Nutrition (EPIC)-Norfolk cohort. Am J Clin Nutr 92, 1214-1222.

39. Patel PS, Sharp SJ, Luben RN, et al. (2009) Association between type of dietary fish and seafood intake and the risk of incident type 2 diabetes: the European prospective investigation of cancer (EPIC)-Norfolk cohort study. Diabetes Care 32, 1857-1863.

40. van Dam RM, Willett WC, Rimm EB, et al. (2002) Dietary fat and meat intake in relation to risk of type 2 diabetes in men. Diabetes Care 25, 417-424. 
41. van Woudenbergh GJ, van Ballegooijen AJ, Kuijsten A, et al. (2009) Eating fish and risk of type 2 diabetes: A populationbased, prospective follow-up study. Diabetes Care 32, 2021-2026.

42. Vang A, Singh PN, Lee JW, et al. (2008) Meats, processed meats, obesity, weight gain and occurrence of diabetes among adults: findings from Adventist Health Studies. Ann Nutr Metab 52, 96-104.

43. Villegas R, Xiang YB, Elasy T, et al. (2011) Fish, shellfish, and long-chain $n-3$ fatty acid consumption and risk of incident type 2 diabetes in middle-aged Chinese men and women. Am J Clin Nutr 94, 543-551.

44. Wang L, Folsom AR, Zheng ZJ, et al. (2003) Plasma fatty acid composition and incidence of diabetes in middle-aged adults: the Atherosclerosis Risk in Communities (ARIC) Study. Am J Clin Nutr 78, 91-98.

45. Micha R, Wallace SK \& Mozaffarian D (2010) Red and processed meat consumption and risk of incident coronary heart disease, stroke, and diabetes mellitus: a systematic review and meta-analysis. Circulation 121, 2271-2283

46. Greenland S \& Longnecker MP (1992) Methods for trend estimation from summarized dose-response data, with applications to meta-analysis. Am J Epidemiol 135, 1301-1309.

47. Orsini N, Bellocco R \& Greenland S (2006) Generalized least squares for trend estimation of summarized dose-response data. Stata Journal 6, 40-57.

48. Micha R, Kalantarian S, Wirojratana P, et al. (2011) Estimating the global and regional burden of suboptimal nutrition on chronic disease: methods and inputs to the analysis. Eur J Clin Nutr

49. Higgins JP, Thompson SG, Deeks JJ, et al. (2003) Measuring inconsistency in meta-analyses. BMJ 327, 557-560.

50. Begg CB \& Mazumdar M (1994) Operating characteristics of a rank correlation test for publication bias. Biometrics 50, 1088-1101.

51. Griffin MD, Sanders TA, Davies IG, et al. (2006) Effects of altering the ratio of dietary $n-6$ to $n-3$ fatty acids on insulin sensitivity, lipoprotein size, and postprandial lipemia in men and postmenopausal women aged $45-70$ y: the OPTILIP Study. Am J Clin Nutr 84, 1290-1298.
52. Giacco R, Cuomo V, Vessby B, et al. (2007) Fish oil, insulin sensitivity, insulin secretion and glucose tolerance in healthy people: is there any effect of fish oil supplementation in relation to the type of background diet and habitual dietary intake of n-6 and n-3 fatty acids? Nutr Metab Cardiovasc Dis 17, 572-580.

53. Egert S, Fobker M, Andersen G, et al. (2008) Effects of dietary alpha-linolenic acid, eicosapentaenoic acid or docosahexaenoic acid on parameters of glucose metabolism in healthy volunteers. Ann Nutr Metab 53, 182-187.

54. Chiu M, Austin PC, Manuel DG, et al. (2011) Deriving ethnic-specific BMI cutoff points for assessing diabetes risk Diabetes Care 34, 1741-1748.

55. Wildman RP, Gu D, Reynolds K, et al. (2004) Appropriate body mass index and waist circumference cutoffs for categorization of overweight and central adiposity among Chinese adults. Am J Clin Nutr 80, 1129-1136.

56. Mozaffarian D \& Wu JH (2011) Omega-3 Fatty Acids and Cardiovascular Disease - Effects on Risk Factors, Molecular Pathways, and Clinical Events. J Am Coll Cardiol 58, 2047-2067.

57. Ghafoorunissa, Ibrahim A \& Natarajan S (2005) Substituting dietary linoleic acid with alpha-linolenic acid improves insulin sensitivity in sucrose fed rats. Biochim Biophys Acta 1733, 67-75.

58. Mustad VA, Demichele S, Huang YS, et al. (2006) Differential effects of $n-3$ polyunsaturated fatty acids on metabolic control and vascular reactivity in the type 2 diabetic ob/ob mouse. Metabolism 55, 1365-1374.

59. Kelley DS, Vemuri M, Adkins Y, et al. (2009) Flaxseed oil prevents trans-10, cis-12-conjugated linoleic acid-induced insulin resistance in mice. BrJ Nutr 101, 701-708.

60. Wendland E, Farmer A, Glasziou P, et al. (2006) Effect of alpha linolenic acid on cardiovascular risk markers: a systematic review. Heart 92, 166-169.

61. Bloedon LT, Balikai S, Chittams J, et al. (2008) Flaxseed and cardiovascular risk factors: results from a double blind, randomized, controlled clinical trial. J Am Coll Nutr 27, $65-74$. 